\title{
LA DISRUPCIÓN DE LO PRESENCIAL A LO VIRTUAL. PERCEPCIONES DE LOS DIRECTORES DE DOCENCIA SOBRE EL USO DE PLATAFORMAS DIGITALES EN CONTEXTO DE PANDEMIA EN UNA UNIVERSIDAD DEL NORTE DE CHILE The Disruption of the Face-to-Face to the Virtual. Perceptions of Teaching Directors on the Use of Digital Platforms in the Context of a Pandemic at a University in Northern Chile
}

Patricio Rivera Olguin ${ }^{1}$

ORCID: 0000-0001-7309-9847

Eugenio Sánchez Espinoza ${ }^{2}$

ORCID: 0000-0002-2902-7296

Beatriz Cortés Díaz ${ }^{3}$

ORCID: 0000-0002-1811-7878

${ }^{12}$ Universidad Arturo Prat, Facultad de Ciencias Humanas, Chile.

${ }^{3}$ Universidad Arturo Prat, Dirección de Armonización Curricular e Innovación Docente, Chile.

Correspondencia: eusanchez@unap.cl

Recibido: 18/06/2021

Aceptado: 22/10/2021

Resumen: Producto de la pandemia de COVID-19, la práctica educativa en los distintos niveles del sistema se vio alterada y sometida a una situación de contingencia que obligó a los docentes a usar herramientas tecnológicas y estrategias para la enseñanza en un contexto de virtualidad. El presente estudio tiene como finalidad describir el paso de una docencia presencial a una educación remota de emergencia y las dificultades que presentó esto para los académicos de la Universidad Arturo Prat (UNAP) de Chile, en cuanto al uso de las tecnologías disponibles para el desarrollo de las actividades lectivas en el contexto de la pandemia. Se desarrolla una investigación de carácter cualitativo, para caracterizar la disposición y problemáticas que evidenciaron los académicos frente a la enseñanza remota, a través de las percepciones de los directores de docencia. Los resultados muestran que los académicos han trasladado el formato de sus clases presenciales a la virtualidad, generando con ello la aplicación de un nuevo formato docente en modalidad remota. Asimismo, se demuestra un uso diferenciado de las tecnologías digitales disponibles según los tramos de edad de los docentes.

Palabras clave: educación remota de emergencia; práctica educativa; educación virtual; educación superior; COVID-19. 
Abstract: As a result of the COVID-19 pandemic, educational practice at different levels of the system was altered and subjected to a contingency situation that forced teachers to use technological tools and strategies for teaching in a virtual context. The purpose of this study is to describe the transition from faceto-face teaching to remote emergency education and the difficulties this presented for academics at the Universidad Arturo Prat (UNAP) in Chile, in terms of the use of available technologies for the development of teaching activities in the context of the pandemic. Qualitative research is developed to characterize the disposition and problems that the academics evidenced regarding remote teaching, through the perceptions of the teaching directors. The results show that academics have transferred the format of their face-to-face classes to virtuality, thus generating the application of a new teaching format in remote modality. Likewise, there is a differentiated use of the available digital technologies according to the age groups of the teachers.

Keywords: remote emergency teaching; educational practice; virtual education; higher education; COVID-19.

\section{Introducción}

En el año 2020, el escenario educativo global sufre una inflexión provocada por la crisis sanitaria mundial producida por el virus SARS-CoV2 (COVID-19) que rápidamente se extiende desde su origen en la ciudad de Wuhan en China (Organización Mundial de la Salud, 2019). En el mundo irrumpe una pandemia que paraliza las actividades de muchos países, donde se confinan a la población para evitar contagios, lo que en Chile sucede en marzo del 2020 (Ministerio de Salud de Chile, 2020).

En este contexto de crisis, la educación suspende sus clases presenciales y aparece un nuevo concepto, el de la educación remota de emergencia (ERE), referenciado por Hodges et al. (2020), que se desarrolla a nivel educativo en sus distintas etapas de formación (Ministerio de Educación de Chile [MINEDUC], 2020). Hodges et al. (2020) definirán básicamente a la ERE como una enseñanza transitoria de respuesta a una crisis.

Las circunstancias sanitarias, que obligaron a millones de personas a permanecer en sus casas, generó que la ERE modificara la disposición del profesorado y estudiantes ante una nueva realidad educativa que ha conllevado diversos desafíos tecnológicos y de docencia nunca antes enfrentados. Dicha situación hace imprescindible conocer cómo este nuevo fenómeno ha afectado los diversos niveles educativos, especialmente en educación superior, puesto que impacta directamente en la formación de futuros profesionales. Los diferentes tipos de clases como laboratorios o prácticas, y la interacción que se daba al interior de estas, cambiaron y se adaptaron para satisfacer las necesidades educativas del momento, quebrando las prácticas tradicionales que hasta ese momento se habían utilizado. 
Sin embargo, no todo se ha abandonado a pesar del cambio de lo presencial a lo virtual, pues existen docentes que simplemente siguen desarrollando su práctica de enseñanza habitual con sus estudiantes, con la sola diferencia de que ahora los separa una pantalla de computador.

De esta forma, este artículo recoge parte de los resultados de una investigación mayor realizada en la Universidad Arturo Prat (UNAP), en el contexto de pandemia y educación remota. El objetivo general es analizar y evaluar las estrategias institucionales implementadas ante la contingencia y cómo se desarrolló la ERE en carreras de pregrado, a partir de la práctica docente virtual, la capacitación en docencia remota y la utilización de plataformas digitales por parte de los docentes de la Universidad. Esta investigación tuvo una amplia cobertura, que involucró académicos, decanos, directivos superiores y estudiantes. Para este artículo se hace foco solamente en las percepciones de los directores de docencia, dado que estos concentran información panorámica de las facultades y las prácticas de sus académicos y docentes.

Los resultados del presente artículo responden a un estudio de corte cualitativo realizado durante el segundo semestre de 2020, respecto del paso de lo presencial a lo virtual. Las preguntas que orientan el desarrollo de este trabajo son: ¿Qué dificultades afrontaron los docentes de la UNAP al transitar de una docencia presencial a una enseñanza virtual y/o ERE? ¿Cuáles son las herramientas digitales utilizadas para la ERE? ¿Cuáles fueron las estrategias pedagógicas para enfrentar la ERE?

En los apartados siguientes se realiza un acercamiento a los conceptos de educación virtual y educación remota de emergencia y se refieren los resultados y conclusiones del estudio, a partir de la sistematización y análisis de las entrevistas realizadas a los directores de docencia de las distintas facultades de la UNAP.

\section{A modo de contexto}

La era digital trajo cambios sustanciales en las formas de trabajo y comunicación, pues se pasó de llevar registros en papel a programas computacionales (software) que permiten gestionar la información de forma más eficiente. Tal situación no demoró en generar nuevas demandas a los sistemas educativos, especialmente en las instituciones de Educación Superior, ya que a los profesionales se les incorporó un requisito adicional: el trabajo con dispositivos y/o herramientas digitales (computador, software y otros). A su vez, esta nueva demanda laboral y sociotecnológica se instauró como una competencia digital (Gutiérrez \& Tyner, 2012; Organización de las Naciones 
Unidas para la Educación, la Ciencia y la Cultura [UNESCO], 2019) que se introdujo progresivamente en los sistemas educativos para luego integrarse plenamente en los currículos a nivel global. Esto llevó al profesor universitario a considerar la incorporación de la tecnología digital en sus clases (Soriano, 2015), lo que derivó en un asunto polémico, dado los cambios que implicaba (por ejemplo, en su práctica y ejercicio docente, técnicas de enseñanza y evaluación, la ausencia de presencialidad, entre otros). Esta situación global propició en algunos países el inicio de proyectos de mejora en la infraestructura tecnológica de las escuelas desde las plataformas digitales al uso de TIC (Jiménez, 2020).

En Chile, el sistema educacional ha invertido elevados recursos económicos en modernizar la infraestructura tecnológica de las escuelas, colegios e instituciones de educación superior del país. Esto ha contribuido a la incorporación de las tecnologías digitales como un instrumento al servicio de la educación de los estudiantes (Bentes et al., 2017). Lo anterior fue acompañado de la transversalización de la competencia digital en la mayoría de los planes de estudio en Chile, a nivel de educación primaria, secundaria y educación superior, donde el rol de los profesores es clave. De esta forma, en el año 2011 se publicaron, por parte del MINEDUC, las Competencias y estándares para la formación docente que contemplan algunas pautas que deben desarrollarse para garantizar el desarrollo de la competencia digital. En paralelo, la mayoría de las universidades de Chile implementaron plataformas tecnológicas que integraron un aula virtual para que los profesores desarrollen un trabajo mixto, entre lo presencial-virtual, y de ser posible a distancia.

Toda esta modernización en infraestructura tecnológica e incorporación de estándares y competencias digitales en los planes de formación se han puesto a prueba a nivel global en el contexto de la pandemia de COVID-19. Las recomendaciones de la OMS para disminuir el contagio y transmisión del virus derivaron en la suspensión de las clases de modo presencial. Tal situación generó que la modalidad de las clases migrara hacia la virtualidad, provocando polémica y tensión en estudiantes y profesorado. Estos últimos, si bien en algún momento habían empleado recursos o dispositivos digitales en sus clases, se vieron igualmente afectados por un traslado total a clases virtuales (Delgado, 2020). Este nuevo escenario virtual demandó una reconfiguración de las génesis en la comunicación (oral, escrita, gestual).

En el caso específico de la UNAP, se disponía de un aula virtual y la plataforma digital Microstf Teams, las que pasaron de herramientas auxiliares o de apoyo a principales. Esta presencia auxiliar y marginal en el uso de plataformas digitales posee una correlación con lo planteado por Buckingham (2006) al decir que en sus inicios estos espacios virtuales parecían no 
impactar en la formación, puesto que los profesores se mostraban escépticos al uso de las tecnologías digitales, es decir, se mantenían indiferentes ante los posibles beneficios educativos de la tecnología. Al respecto, se debe señalar y recordar que una de las demandas más exigidas en los sistemas educativos es la competencia digital profesional (Pardo \& Cobo, 2020), dada su baja presencia en los profesionales de este ámbito (Sierra et al., 2018), y que producto de la pandemia se reveló como auténtica necesidad dada la ausencia de capacitación (Palacios \& Barroso, 2020).

\section{Docencia remota de emergencia}

Gran parte de los docentes chilenos, en diversos niveles, debieron adaptarse a la situación que implicó realizar sus actividades lectivas a través de una plataforma digital. El impacto fue alto y los costos aún se están evaluando, sobre todo, desde la perspectiva del nivel de aprendizaje de los estudiantes. ${ }^{1}$

La ERE se transformó en la única alternativa viable para mantener los lazos entre las instituciones educativas, sus docentes y estudiantes. Los esfuerzos han sido enormes y variados con la finalidad de llegar a todos los estudiantes sin importar su condición socioeconómica. Lo anterior es relevante, pues en este punto se tornó clave el acceso a Internet y a equipos tecnológicos que soportaran de la mejor manera una plataforma que permitiera dar algún grado de normalidad a la actividad educativa.

Por tanto, este formato es una respuesta transitoria a una crisis, donde "la enseñanza remota de emergencia es un cambio temporal de la entrega de instrucción a un modo de entrega alternativo debido a circunstancias de crisis" (Hodges et al., 2020, p. 17). En relación con la transitoriedad, se plantea que las soluciones de "enseñanza aplicadas al campo educativo, que de otro modo se impartirían presencialmente o como cursos combinados o híbridos, volverán a ese formato una vez que la crisis o la emergencia hayan disminuido" (p. 17).

Esta cobertura pedagógica se implementa en el sistema de educación superior, en condición de confinamiento, al entregar la red de contenidos y pasar las clases de la presencialidad a la virtualidad mediante diversas plataformas de soporte (Cea et al., 2020). Sin embargo, en el proceso de ajuste surgen distintas complejidades, quedando en evidencia que "el desafío docente fue

\footnotetext{
${ }^{1}$ Es interesante señalar que los posibles impactos de la modalidad remota y efectos de la práctica de los académicos sobre el desarrollo de aprendizajes y logro de competencias en los estudiantes, en el contexto de la pandemia, será evidenciado de forma específica en una futura publicación que forma parte de la línea de investigación desarrollada en la UNAP.
} 
adaptar las asignaturas presenciales al aprendizaje remoto de emergencia” (Pardo \& Cobo, 2020, p. 23) según cada contexto educativo.

Al respecto Sangrà (2020) es realista al señalar que nadie espera recetas para afrontar esta situación tan particular, debido a que la crisis es manejada de "manera distinta, en función de su contexto social y económico, de las edades de sus estudiantes, de las regulaciones nacionales, por lo que es enormemente arriesgado pretender dar recetas" (p. 27).

Las múltiples realidades y contextos educativos producen diferentes elementos de discusión sobre la definición de la ERE. Por ejemplo, en algunos contextos se asume que no es una enseñanza virtual, mas el concepto se discute en todas las latitudes y a todo nivel del sistema educativo. Por otra parte, Pardo \& Cobo (2020) indican que

\begin{abstract}
Muchas universidades han improvisado, llegando tarde o con una preparación insuficiente a esta adaptación, aplicando voluntarismo y replicando en la virtualidad modelos de aprendizaje que ya están siendo obsoletos en la presencialidad. Más allá de algunas buenas prácticas de docentes y equipos de desarrolladores, la pandemia demuestra que la mayoría de las universidades de Iberoamérica están en vías de desarrollo en cuanto a lo digital” (p. 8).
\end{abstract}

Asimismo, Sangrà (2020) plantea que es la interacción con el estudiante lo que distingue cada modelo pedagógico online: "no confundaís interacción con comunicación sincrónica. La interacción se puede llevar a cabo en distintos formatos y soportes, pero no puede hacerse igual que presencialmente, porque entonces probablemente fracasaremos" (Sangrà, 2020, p. 35).

Ahora bien, la definición de ERE signada por Hodges et al. (2020) aporta mayor claridad al debate cuando indican que esta no busca ser un modelo, sino que es un formato de tránsito y de respuesta a contextos críticos, distinta de los aprendizajes en línea o e-learning. Los autores manifiestan de manera más concreta que:

El objetivo principal en estas circunstancias no es recrear un ecosistema educativo robusto, sino más bien proporcionar acceso temporal a la instrucción y a los apoyos instructivos de una manera rápida y fácil de instalar durante una emergencia o crisis. Cuando entendemos la ERE de esta manera, podemos comenzar a divorciarlo o distanciarnos del "aprendizaje en línea" (Hodges et al., 2020, p. 17).

Por tanto, es clave que en este debate se delimiten y clarifiquen los conceptos respecto de uno u otro formato o modelo de educación no presencial, que puede ser variado en los distintos niveles de formación e ir desde lo general hasta lo más específico (Goodyear, 2005). 
Respecto de los modelos de formación presenciales o mixtos, es posible mencionar que se fundan en perspectivas de educación disímiles, tanto en sus enfoques como en sus teorías (López \& López, 2013). Esta diferenciación se ha trasladado a la modalidad virtual debiéndose considerar algunos aspectos de interés, ya que desde la docencia se enuncian diferentes modelos pedagógicos en el campo de los aprendizajes en las TIC.

A modo de ejemplo, Stephenson y Sangrà (s.f.) revelan la existencia de modelos transmisivos, de aprendizaje por la práctica y los colaborativos, y plantean que los e-learning se pueden centrar más en aprendizajes que en la enseñanza.

Además, se debe señalar que la adaptación del modelo presencial se manifiesta en la ERE, dado que el uso de las TIC se basa en modelos clásicos como forma de consolidar las acciones que promuevan el aprendizaje en línea. Al respecto, Maina (2020), citando a Ron Oston, esboza para las clases sincrónicas los siguientes modelos: modelo interactivo equilibrado, modelo de seminario, modelo de video interactivo y modelo basado en contenidos que podrían ser modelos de formación en la DRE.

A su vez, estos modelos deben estar conectados con estrategias, y Romeau (2020) destaca tres: 1) incorporación de metodologías activo-participativas; 2) integración de la competencia digital docente y 3) presencia docente en los entornos de aprendizaje en línea. Incorporar estos tres elementos permitiría alcanzar un cambio transcendental, donde Badia (2020) destaca que "el profesorado probablemente también tiene que modificar su visión sobre la función de la tecnología en los entornos online de aprendizaje" (p. 133).

Después de todo, recordemos que la temática de la virtualidad tiene su origen en los modelos e-learning, que pueden ser considerados como un catalizador positivo de los modelos pedagógicos que se impulsan actualmente (Stephenson y Sangrà, s.f.). Por ejemplo, los autores recién referidos demuestran que un modelo integrado busca "la flexibilidad, la personalización, la interacción y la cooperación, permitiendo que sea el estudiante quien lo gestiona todo a su conveniencia, mientras la institución, establece medidas de garantía y control en la validación de los conocimientos adquiridos" (p. 10).

De la misma forma, manifiestan que la modalidad e-learning no tiene adscripción a un modelo pedagógico en particular y afirman que "el e-learning es cualquier medio electrónico de distribución, participación y apoyo al aprendizaje, normalmente mediante internet y de servicios de medios electrónicos, relacionados como al aprendizaje por ordenador, las aulas virtuales y la colaboración digital” (p. 13). 
También vale citar a Rodríguez (2020) que advierte que:

Los entornos virtuales de aprendizaje son un claro reflejo de los cambios en el paradigma docente. Así, el centro de estos entornos son los propios alumnos, que adquieren un rol activo en su propio proceso de aprendizaje. Mientras que el docente abandona su tradicional rol protagonista para adquirir el rol de facilitador del proceso de aprendizaje, asegurándose de preparar adecuadamente todo el entorno para que el alumno pueda alcanzar las competencias establecidas (p. 20).

Sobre este aspecto, la ERE se instaló en medio de un escenario de incertidumbre donde la enseñanza virtual se ha desplegado requiriendo saberes tecnológicos, mientras los "profesores cuando desarrollan enfoques de e-learning por primera vez, reproducen su enfoque presencial habitual en lugar de explotar las posibilidades que ofrece la tecnología" (Stephenson y Sangrà, s.f., p. 19).

En síntesis, un acontecimiento sanitario complejo a nivel mundial ha dejado al descubierto de forma palpable una situación precaria en cuanto la capacidad de los docentes para hacer uso de la tecnología. Esto demuestra que su integración al aula y su uso, en general, sigue siendo más rudimentario que efectivo, lo que afecta probablemente el desarrollo de aprendizajes.

\section{Metodología}

El carácter cualitativo de la metodología empleada tuvo por finalidad explorar cómo se desarrolló el paso de una práctica docente presencial a un contexto de docencia remota de emergencia en educación superior, específicamente en el caso de la UNAP. Asimismo, el carácter de la investigación exigió el reconocimiento de múltiples realidades (a nivel de facultades, por ejemplo) y trató de capturar la perspectiva, en este caso, de los informantes clave.

\section{Muestra y criterios}

Estos informantes clave son la totalidad de los directores de docencia de las seis Facultades de la UNAP quienes, además de dictar clases, son los encargados de la correcta aplicación del Modelo Educativo Institucional, de la gestión docente y buen desempeño de los académicos para asegurar la calidad de la docencia. En consecuencia, sus participaciones pueden entregar información precisa sobre las dificultades y manejo de las tecnologías para la docencia remota. Por lo tanto, primó el parámetro de significatividad y relevancia de la información. Los 
entrevistados son identificados con la sigla DDF, que hace referencia al cargo de dirección de docencia de facultad.

\section{Técnica de recolección de información}

Para la obtención de antecedentes se utilizó la entrevista en profundidad, pues permite acceder a información para el análisis de las opiniones, conocimientos, prácticas y dificultades que enfrentaron los docentes en la implementación de la ERE. Las entrevistas fueron aplicadas de forma flexible y virtual, el segundo semestre del año 2020, a partir de una serie de preguntas organizadas en dimensiones previamente establecidas y vinculadas al uso de plataformas, herramientas digitales y docencia virtual y remota.

\section{Análisis}

Una vez transcritas las intervenciones de los participantes, se establecieron las unidades de análisis relativas a los segmentos que interesaba indagar para, consecutivamente, traducirlas y desglosarlas en categorías. En este sentido, se identificaron como categorías de análisis centrales las dimensiones pedagógica, para ahondar en aspectos curriculares, metodológicos y evaluativos,

y la de gestión y desarrollo, vinculada a acciones definidas por la institución junto a su seguimiento y progreso.

\section{Resultados y discusión}

La UNAP comienza el confinamiento el 15 de marzo del 2020 y decide, desde su comité de crisis, iniciar la modalidad de clases virtuales, que a partir de los antecedentes ya desplegados se puede identificar y contextualizar como ERE (Hodges et al., 2020). Por tanto, la docencia virtual fue la cotidianidad de la comunidad universitaria. En este escenario se desarrolló un proceso de adaptación que cambió el aula presencial por una plataforma virtual.

En este sentido, la UNAP centró su formación de pregrado en dos plataformas: un aula virtual (AV, con software de código abierto Sakai Project) y Microsoft Teams de Windows. La primera plataforma data del año 2014 y su uso por parte de los académicos siempre fue más bien distante y esporádico. Mientras que Microsoft Teams se pone a disposición de la comunidad educativa en marzo del 2020, desarrollando en paralelo períodos de capacitaciones a los académicos a manos de la Unidad de Virtualización. 


\section{Dimensión pedagógica}

Las comunicaciones que tienen los cuerpos académicos es canalizada por las direcciones de docencia, por ello fue fundamental su labor en los procesos de virtualización al igual que su percepción y apreciación de este proceso. En este contexto se pudieron registrar las fortalezas de la ERE, la participación de los académicos en las capacitaciones para formarlos en el uso de los sistemas online y la participación activa para poder desarrollar sus clases sin dificultades.

Ahora bien, la virtualidad no fue ajena para algunos académicos, dado que ya poseían cierta experiencia, destacándose que la instalación de las plataformas contribuyó a su proceso de formación. Así, para algunos colegas, "la virtualización no es un problema, porque viene a ser una solución; porque tenemos mucho trabajo de campo, viene a ser una solución maravillosa por la sincronía de clases" (DDF2).

De igual forma, en los contenidos de las entrevistas a los DDF existe un constante reconocimiento a la labor académica, centrado en el compromiso y la disponibilidad para la implementación de la virtualidad manifestado desde un escenario prepandemia en el 2019.

Existe un compromiso, porque fue difícil el cambio, cuando se vio que era para extenso el tema de la virtualidad, hubo harto compromiso de parte de los docentes en participar, creo que una de las fortalezas fue el compromiso e interés para aprender para poder aplicarlo de la mejor manera (DDF2).

También es interesante apreciar una adaptación al sistema, dada la particularidad de las plataformas, porque se asimilan al aula presencial generando mayor comodidad.

Yo diría que se adaptan mejor a Teams, porque es lo que más les resulta más familiar; pero Teams, ya sea sincrónico o asincrónico, es más fácil para los profesores para hacer una clase (DDF5).

Esta cercanía se evidencia cuando los académicos asimilan la plataforma, puesto que "en el Teams, es la relatoría, es llegar y dictar una clase" (DDF5).

Sin embargo, esto que al inicio parecía sencillo y perfecto comenzó a presentar ciertos cuestionamientos vinculados a la efectividad de realizar clases netamente expositivitas por esta vía. El esfuerzo docente en instalación de la ERE es algo que se debe destacar y se aprecia en la constancia por continuar asumiendo un rol docente tecnológico que no se tenía y desarrollando una solidaridad académica para desplegar alternativas de trabajo en forma colaborativa. 
Yo creo que la mayor fortaleza es que [los docentes] sí o sí, ingresaron en esta estructura de virtualidad y se formaron equipos dentro de las facultades, entre colegas, y el que más sabía más ayudaba al que sabía menos y entre todos fuimos construyendo para que las clases salieran de la mejor forma posible (DDF6).

A la vez, se instala la necesidad de la capacitación como iniciativa fundada en un trabajo constante y colaborativo como una manera de desarrollar y potenciar las actividades que propiciaban los académicos.

Una de las estrategias que consideramos fue la capacitación, porque en el fondo es realizar las clases presenciales de manera virtual y significa también cambiar un poco el paradigma, hacia un desarrollo asincrónico y más autónomo, entonces conversamos, incluso en unas asambleas al inicio del semestre y conversamos en el interior del equipo en hacer una capacitación el aspecto virtual en forma más empoderada. (DDF1).

Por otra parte, el desarrollo de la ERE, también dejo en evidencia las brechas en las capacidades tecnológicas del cuerpo académico, ya que "hay cierta rigidez para poder utilizar las herramientas, porque después de hacer una clase, tiene que pasarse al sistema y deben ponerse obligadamente las evaluaciones, lo que era complejo para algunos colegas al no manejar el aula virtual" (DDF1).

Este proceso de adaptación fue complejo y necesitó tiempo, dado que la ERE debió ser desarrollada por académicos con distinto nivel en cuanto manejo de tecnologías, y sobre todo cuando hicieron frente al cambio vertiginoso de un formato presencial a otro virtual.

El período de adaptación fue rápido, a pesar de que no estoy lejos del grupo etario, como podrían estar otros colegas; fue complejo, yo me di cuenta de un porrazo de aquello, el subir ciertas evaluaciones, el grabarme haciendo clases en la pizarra, no era algo que los estudiantes apreciaran, ósea apreciaban el esfuerzo, no apreciaban el resultado, me lo hicieron saber, incluso por correo electrónico (DDF2).

A su vez, se debe destacar que el uso de la plataforma Teams era asimétrico en los académicos, requiriendo capacitaciones al iniciarse la ERE. Los registros señalan que el rango etario fue el factor más influyente.

Teams es una plataforma que llevaba años en la institución, yo trabajé con ella en el programa Trabajador, pero en lo general los docentes de la universidad, están muy nulos con todos los ambientes y los temas virtuales de aprendizajes, ya que, en aula virtual, lo único que sabían era subir material y nada más y de repente los más jóvenes, teníamos más llegada a la tecnología y nos metíamos un poco más (DDF3). 
Otro elemento propio de los procesos de cambio y adaptación que surgió fue la resistencia; más aún en un contexto imperativo de una docencia remota que alteró la tradicional presencialidad.

Por la resistencia al cambio, muchos colegas se rehúsan en crear programas de educación virtual o e-learning, que tenga una mezcla de dos cosas, fuimos probando hartas TICS de hecho somos súper pocos los que hacemos clases con tablets y sirve para hacer ejercicios, hacer preguntas, test con herramientas como Kahoot (DDF3).

En esa lógica de acción con dificultades, los académicos se activan frente a esta nueva docencia mediante innovaciones como cápsulas que tropiezan con las complejidades técnicas de la plataforma coartando las iniciativas docentes.

Yo, por ejemplo, grabo mis cápsulas cotas, 5, 10 minutos, pero cada vez que cargas la página, se demora mucho, se corren los videos, todos los audios y queda como ensalada, pero no puedo mostrarle eso a los estudiantes, lo cargo como un archivo no más (DDF3).

Los académicos también reconocen una debilidad en las metodologías de enseñanza, que se traducen en un estancamiento que provoca aburrimiento en los estudiantes debido a la reiterada exposición, que produce un cuestionamiento a los enfoques y el desarrollo de competencias.

Esto de la pandemia dejó claro que está fallando la metodología que ocupamos, me incluyo, pero también, deja en evidencia a los estudiantes y fallan las cosas, una que el profesor usa metodología muy aburrida, la clásica, tres pruebas, teoría y la clase expositiva. Lo más lúdico es ocupar una diapositiva de power point y que tenga alguna fotito (DDF2).

Además de los diversos obstáculos planteados por el escenario de las clases virtuales, emergen nuevos conceptos como sincronía y asincronía, que marcan la relación de los estudiantes con este proceso de docencia remota y sus docentes.

Los alumnos se resisten, la hora sincrónica es obligatoria y la hora indirecta o asincrónica no la usan. Entonces este modelo pedagógico virtual, no sirve. En cambio, cuando estamos en aula [presencial]aprende más y ahora como se aburren y tienen el computador, pueden abrir Facebook y no está dando resultado con subir material asincrónico para que se logre el aprendizaje (DDF2).

Los profesores también cuestionaron el enfoque de enseñanza, dado que, al existir mínimas capacitaciones, no consiguen el logro de los aprendizajes dejando en evidencia una postura crítica de los enfoques en educación superior. Manifiestan, por ejemplo, que es muy complejo aplicar el 
modelo por competencias declarado y que no existen capacitaciones específicas al respecto, ni tampoco quien evalúe técnicamente el logro de objetivos y nivel de las competencias.

La homologación de las plataformas a la presencialidad es indicio de la permanencia de la práctica docente tradicional que busca el restablecimiento del aula presencial. Lo anterior, no permite mayor y mejor uso de la movilidad de los formatos de las clases virtuales, ni tampoco genera mayor interacción con los estudiantes.

Yo diría que se adaptan mejor a Teams, porque se asemeja al aula presencial y se adaptan menos a otras fórmulas en que existiría un proceso de calibración, como un foro; ahora en el Teams, no te mueves mucho, si es asincrónico, no tiene relación con los estudiantes y si es sincrónico, tienes solo pantallas apagadas, entonces no los vemos ni las caras y se pierde ese tiempo de interacción (DDF5).

El contexto de emergencia creó un escenario nuevo para el cual no se estaba preparado y eso llevó a una planificación distinta a la tradicional. Por tanto, la virtualidad se erigió en la incertidumbre y la gestión no alcanzó a responder en forma efectiva.

Yo creo que el principal problema, fue responder desde una planificación que era la normal, la presencial. Entonces, lo principal desde la gestión, era improvisar una planificación virtual con cierta incertidumbre, ahora no sabemos si esta forma es la adecuada y si están aprendiendo nuestros estudiantes (DDF5).

También es necesario indicar que existe una percepción académica diferida respecto a las plataformas, dado que el Teams es cercano a las clases presenciales, en tanto el aula virtual es un ámbito que se percibe como más tecnologizado y esto se debe a que alberga múltiples herramientas virtuales. Esto último, se traduce se traduce en un foco de debilidad que amerita capacitaciones.

El Teams es la relatoría y el aula virtual se ocupa mucho el sistema de mensajería, para enviar tarea y para que devuelvan tarea, hay foro, yo por lo particular ocupo el foro, porque tengo que plantear un tema, tampoco doy tarea, porque todavía no logro captar de qué forma lo uso con los estudiantes, lo mismo que el examen, por eso yo digo que allí hay falta de capacitación (DDR6).

Asimismo, el sentido de la clase remota preocupa a los docentes, que cuestionan la forma en que se realiza junto al impacto que tiene en los estudiantes. Al respecto manifiestan que es imposible "tener al estudiante entretenido dos horas frente al computador, entonces ha sido complicado. Yo recuerdo la primera clase y no lleva a ningún lado, hay profesores que se resistieron porque no están acostumbrados a una videoconferencia” (DDF4). Aquí se observa cómo se ve afectado el proceso comunicativo, ya que no se desarrolla una interacción entre el 
docente y el estudiante y, por ende, se ve disminuida la comprensión y la retroalimentación que debe existir en una clase.

Finalmente, el contexto de emergencia posiciona a la virtualidad como formato de docencia y entrega a las plataformas digitales, ya existentes en la Universidad, la función y centralidad para las que fueron implementadas antes de la pandemia. Esto, en este contexto emergente, también permitió que ellas ya no solo fueran vistas y utilizadas como un repositorio donde almacenar y transferir información, sino como un elemento que se hace parte de su práctica educativa.

\section{Dimensión de gestión y desarrollo}

En esta dimensión se ahonda en los espacios de decisión que cada Facultad tiene por su especialidad o perfil de formación, dado que emergieron distintas estrategias desde el cuerpo académico o de los propios equipos encargados de esta área. En este sentido, era necesario conocer si existían acciones para el apoyo de la docencia remota.

Así pues, se consideró fundamental desarrollar capacitaciones desde las facultades, a la vez que resulta muy interesante un cambio de la visión sobre la formación, e incluso del paradigma educativo, por la existencia de una necesaria y obligada reflexión del quehacer docente que define nuevas formas de evaluación y retroalimentación. Ello queda refrendado cuando se indica que:

Una de las estrategias que consideramos es la capacitación, dado el entorno que es virtual y que significa también cambiar un poco el paradigma y cambiar el sentido hacia un desarrollo más asincrónico y autónomo con una mirada a una retroalimentación del docente con el estudiante (DDF1).

Asimismo, y si bien la situación es de complejidad, se impone un criterio de priorización de acciones institucionales que demuestra una postura institucionalizada hacia el quehacer administrativo, por sobre una estrategia formativa ante la emergencia. Por ejemplo, se señala: "no da pensar en eso, estamos pensando en otras cosas, como la certificación para la acreditación, la reestructuración de la universidad, etc. Entonces, por lo menos, yo no le he pensado" (DDF5).

Estas reacciones manifiestan una débil autorregulación organizacional efectiva ante problemáticas emergentes, y vienen retardar los procesos de mejoramiento en la calidad de los procesos de formación.

Sin embargo, ante la urgente necesidad de implementar una ERE efectiva, se desarrollaron iniciativas en la gestión académica a nivel de facultades y carreras para afrontar el escenario remoto de enseñanza. Lo anterior es apreciable en la siguiente intervención: 
Con el Plan de Implementación se ha considerado al año 2021 de 10 laboratorios móviles por carrera para los docentes puedan ocupar los computadores, pero con el fin de que, si vuelve a pasar algo, similar los estudiantes puedan llevar a su casa los equipos y poder trabajar desde la virtualidad. En cuanto al área docente, estamos trabajando para una capacitación para desarrollar nuevas herramientas virtuales (DDF1).

Este escenario de ERE ha generado interpretaciones al plan institucional, por tanto, se necesita una conexión de los planes que emergen desde la base académica hasta la toma de decisiones institucionales.

Claramente con todo lo que está pasando, el nuevo plan de desarrollo va a apuntar a eso, lo hemos conversado, no se ha creado todavía, porque queremos esperar el institucional, ese el que nos da el paraguas a todas las facultades y desde allí proyectar a la nuestra, pero sin duda que va a ser la temática del entorno virtual (DDF4).

Paralelamente, el tema de las metodologías en entornos virtuales pasa a ser una necesidad que se debe subsanar y con ello potenciar las actividades que se están implementando en ERE, como el uso de biblioteca digital y las bases de datos. Al respecto, se manifiesta que:

Hemos estado trabajando en la planificación, ya proyectamos que el entorno virtual va a ser parte de nuestro trabajo, entonces va a implicar capacitación, mejora de la biblioteca digital, porque ahora que estamos en cuarentena, no pueden ir a buscar libros, va a requerir inversión entre la virtualidad y la presencialidad en la etapa posterior, tuvimos una jornada triestamental, donde se evaluó el entorno virtual y tenemos varios puntos de vista: administrativo, docentes y estudiantes, nos va a servir como insumo ante el proceso virtual y como determinar acciones (DDF4).

La referencia a la institución en coherencia a planes de acción es manifiesta, notándose una intencionalidad de actuar en conjunto frente al escenario de emergencia, sobre todo en metodologías de enseñanza con las unidades especializadas de docencia de la institución.

Los planes era pensados para el contexto presencial, pero nunca pensé que esto de la virtualización se extendería mucho más, así que no se consideró en el 2020 y para el 2021, nos hemos reunido, para hablar qué hacer, medidas como autoaprendizaje o metodologías con las direcciones de docencia de la universidad (DDF5).

Dado el análisis de los registros, es posible advertir que las facultades tienen una visión particular, según la orientación de las carreras que las componen, es por ello que, frente al apoyo a la docencia remota y el aprendizaje, poseen variadas formas de interpretar este proceso. 
También se evidenció que la evaluación docente podría arrojar resultados para la toma de decisiones y entregar indicadores que consideren el actual desarrollo de la docencia remota. Por otra parte, no se debe olvidar el tema de la conectividad y de acceso a equipos por parte de los estudiantes como un factor que inhibe el desarrollo de la ERE. Ambos elementos se encuentran interrelacionados, como se aprecia a continuación.

\begin{abstract}
Ahora la evaluación viene precisamente orientada a cómo el docente, está desarrollando sus actividades virtuales y realizar algún tipo de indicador, porque no tenemos y los estudiantes lo están manifestando. Aún no tenemos todas las condiciones de conectividad para todos los estudiantes y sí la universidad entregó un chip, no fue posible de usar porque hay estudiantes en zonas rurales o porque viven en lugares que no captan la señal. La mayoría lo está haciendo de sus celulares o de tablets, unos pocos con computadores (DDF1).
\end{abstract}

Es interesante examinar que la propuesta de levantar indicadores que permitan analizar la efectividad de las clases virtuales surja como una forma de estrategia, reactiva ante la emergencia.

Finalmente, en los argumentos de los entrevistados se hace evidente la existencia de una constancia de reconocimiento a la labor académica, centrada en el compromiso y la disponibilidad para la instalación de la virtualidad y que es constatable en el interés registrado desde un escenario prepandemia en el 2019.

\title{
Conclusión
}

Los registros de los académicos consultados, todos ellos DDF, demuestran un panorama donde las clases presenciales son replicadas en la enseñanza remota o ERE, revelada en la intencionalidad y metodología expositiva desarrollada en las aulas virtuales. Con lo anterior, se puede considerar que existe una práctica docente anclada aún en la clase frontal, que se consolida con el uso de la plataforma Microsotf Teams. En cambio, el aula virtual es mínimamente implementada, dado que posee una mayor cantidad de herramientas interactivas, lo que lleva a los académicos que reproducen el enfoque presencial a no usar mayormente la tecnología digital disponible en dicha plataforma.

Asimismo, la flexibilidad curricular en los contenidos, cobró mayor importancia en un contexto incierto producto de la pandemia y sobre lo cual es necesario profundizar, pues se esgrimen razones contextuales de sintetizar los programas de asignaturas, lo cual puede considerar futuros rediseños curriculares. 
A la vez, se registra una asimetría en el desarrollo de la ERE, que se evidencia principalmente en el manejo de tecnologías digitales por los académicos. Es un dato relevante que puede explicarse por el rango etario de estos al estar más cercanos o lejanos al uso digital, generando mayor o menor despliegue de clases y posibles innovaciones. Además, se destaca también que existe una omisión relativa a las evaluaciones, lo que se traduce en un punto ciego en la ERE, dado que no hay referencia o visibilización de estas. El resultado es la ausencia de un modelo de clase virtual, dejando en al descubierto la improvisación de la docencia universitaria por la crisis sanitaria.

También, es muy interesante notar que no hay manejo o conocimiento del término ERE, sino que se visualiza como educación virtual, por lo cual se infiere que no hay mayores saberes o consultas a bibliografía especializada del tema, generando un problema de actualización de un tema contingente, que pasa a constituirse en un obstáculo para la toma de decisiones y mejoramiento de la práctica docente y procesos de formación.

A su vez, la retroalimentación como recurso de monitoreo y flujo de conocimientos es poco referida. Ella no es desarrollada, perdiéndose la oportunidad de potenciar un eficaz elemento recursivo en ERE y alcanzar logros efectivos en materia de formación y educación superior. Lo anterior tiene como correlato la ausencia de un modelo guía en la docencia remota que, dada esta característica, sería un modelo transmisivo basado en la práctica.

Igualmente, es concluyente una resistencia a la ERE y sobre todo a la innovación en virtualidad, dado factores como agobio laboral y deficiencias en manejo de TIC, siendo necesario que el profesorado genere un cambio al momento de desarrollar una docencia virtual que modifique su visión de los entornos virtuales y de tecnología digital.

También fue posible constatar un cuestionamiento al modelo de enfoques en competencias, que lleva a reconsiderar el modelo educativo institucional, como resultado del proceso reflexivo que se ha realizado respecto de cómo se ha desarrollado la docencia remota. Esta reacción es comprensible, dado que los modelos e-learnirng son catalizadores de los procesos pedagógicos.

Finalmente, se acentúa que en el escenario postpandemia, dada la relevancia adquirida por las TIC, se cuestione la instalación de la ERE en educación superior, porque se realizó de forma improvisada en contexto de emergencia. De ahí que sería más atingente considerar dicho escenario como algo nuevo, dinámico y transformador de prácticas, por cuanto las TIC dejaron de ser aquel elemento accesorio de prepandemia. Hoy ha quedado demostrado su papel estratégico y esencial en el ejercicio de la docencia en estos nuevos contextos. 


\section{Referencias}

Badia, A. (2020). La mediación pedagógica y tecnológica para el desarrollo de competencias. En A. Sangrà (Coord.), Decálogo para la mejora de la docencia online. Propuestas para educar en contextos presenciales discontinuos (pp. 138-149). Editorial UOC.

Bentes, J., Ortiz, L., \& Cuadra, F. (2017). Las TIC en la integración curricular transversal del quehacer educativo. $\begin{array}{llll}\text { Conhecimento } \quad \& \quad \text { Diversidade, } & \text { 48-60. }\end{array}$ https://revistas.unilasalle.edu.br/index.php/conhecimento_diversidade/article/view/3736

Buckingham, D. (2006). La educación para los medios en la era de la tecnología digital. Trabajo presentado en el congreso del décimo aniversario de MED La sapienza di comunicare. Roma, Italia.

Cea, F., García Hormazábal, R. A., Turra Chico, H., Moya Figueroa, B., Sanhueza Jara, S., Moya Sobarzo, R., \& Vidal Hernández, W. A. (8 de junio de 2020). Educación online de emergencia: Hablando a pantallas en negro. CIPER-Chile. https://www.ciperchile.cl/2020/06/08/educacion-online-de-emergencia-hablando-apantallas-en-negro/

Delgado, K. (2020). La conectividad como recurso de aprendizaje durante la emergencia en los países de América Latina. En I. Aznar, M. Cáceres, J. Marín, \& A. Moreno (Coords.), Desafíos de la investigación educativa durante la pandemia Covid-19 (pp. 70-77). Editorial Dykinson.

Goodyear, P. (2005). Educational design and networked learning: Patterns, pattern languages and design practice. Australasian journal of educational technology, 21(1), 82-101. https://doi.org/10.14742/ajet.1344

Gutiérrez, A., \& Tyner, K. (2012). Educación para los medios, alfabetización mediática y competencia digital. Comunicar, 19(38), 31-39. https://doi.org/10.3916/C38-2012-02-03

Hodges, C., Moore, S., Lockee, B., Trust, T., \& Bond, A. (2020). La diferencia entre la enseñanza remota de emergencia y el aprendizaje en línea. En A. Cabrales, A. Graham, P. Sahlberg, C. Hodges, S. Moore, B. Lockee, T. Trust, A. Bond, D. Lederman, J. Greene, J. Maggioncalda, L. Soares, G. Veletsianos \& J. Zimmerman (Coords.), Enseñanza de emergencia a distancia: textos para la discusión. (pp. 12-22). The Learning Factor.

Jiménez, I. (2020). Rasgos y tendencias de la Didáctica con TIC: retos a partir de la nueva ecología del aprendizaje. Estudios Pedagógicos, 46(2), 215-229. https://doi.org/10.4067/S0718-07052020000200215

López, M., \& López, A. (2013). Los enfoques de aprendizaje, revisión conceptual y de investigación. Revista Colombiana de Educación, 64, 131-153. https://doi.org/10.17227/01203916.64rce131.153

Maina, M. (2020). E-actividades para un aprendizaje activo. En A. Sangrà (Coord.), Decálogo para la mejora de la docencia online. Propuestas para educar en contextos presenciales discontinuos (pp. 81-98). Editorial UOC.

Ministerio de Educación de Chile. (2020). Mineduc lanza nueva oferta formativa para apoyar a la comunidad docente en el contexto de emergencia sanitaria. https://www.mineduc.cl/desarrollo-docente-en-linea/

Ministerio de Salud de Chile. (2020). Plan de acción Corona virus Covid 19. http://epi.minsal.cl/plan-de-accioncoronavirus-2019-ncov/

Organización Mundial de la Salud. (2019). Brote de enfermedad por coronavirus (COVID-19). https://www.who.int/es/emergencies/diseases/novel-coronavirus- 
2019?gclid=CjwKCAiAu8SABhAxEiwAsodSZNX2YF0X3dggMvYEpLEaKgPymQzifZuFKgZMdOCrtp 1rzDQxo5uE2RoCe9YQAvD_BwE

Palacios, A., \& Barroso, J. (2020). Innovación y uso de la tecnología digital. Estudio de la Competencia digital docente. En I. Aznar, M. Cáceres, J. Marín, \& A. Moreno (Coords.), Desafíos de la investigación educativa durante la pandemia Covid-19 (pp. 196-206). Editorial Dykinson.

Pardo, H., \& Cobo, C. (2020). Expandir la Universidad más allá de la enseñanza remota de emergencia. Ideas hacia un modelo híbrido post-pandemia. Outliers School.

Rodríguez, B. (2020). Entornos virtuales de aprendizaje y aprendizaje colaborativo en una sociedad que avanza. En B. Rodríguez (Comp.), Docencia Colaborativa Universitaria: planificar, gestionar y evaluar con los entornos virtuales de aprendizaje. Ediciones de la Universidad Castilla de la Mancha.

Romeau, T. (2020). Cinco estrategias claves para la docencia en línea. En A. Sangrà (Coord.), Decálogo para la mejora de la docencia online. Propuestas para educar en contextos presenciales discontinuos (pp. 138-149). Editorial UOC.

Sangrà, A. (Coord.). (2020). Decálogo para la mejora de la docencia online. Propuestas para educar en contextos presenciales discontinuos. Editorial UOC.

Sierra, J., Romero, B., \& Palmezano, Y. (2018). Causas que determinan las dificultades de la incorporación de las TICS en las Aulas de Clases. Revista Panorama, 12(22), 32-41. https://doi.org/10.15765/pnrm.v12i22.1064

Soriano, I. (2015). El Ser Docente digital en el Siglo XXI. ¿Hacia dónde vamos?. Revista Educación y Tecnología de la Sociedad Mexicana de Computación en la Educación (SOMECE),3(8), 46-66.

Stephenson, J., \& Sangrá, A. (s.f.). Fundamentos del diseño técnico-pedagógico en elearning. Modelos pedagógicos y e-learning. Editorial UOC.

Organización de las Naciones Unidas para la Educación, la Ciencia y la Cultura. (2019). Marco de competencias de los docentes en materia de TIC (Versión 3). https://unesdoc.unesco.org/ark:/48223/pf0000371024

\section{Cómo citar}

Rivera Olguin, P., Sánchez Espinoza, E., \& Cortés Díaz, B. (2021). La disrupción de lo presencial a lo virtual. Percepciones de los directores de docencia sobre el uso de plataformas digitales en contexto de pandemia en una Universidad del norte de Chile. Páginas de Educación, 14(2), 77-95. https://doi.org/10.22235/pe.v14i2.2607

\section{Contribución autoral}

a) Concepción y diseño del trabajo; b) Adquisición de datos; c) Análisis e interpretación de datos; d) Redacción del manuscrito; e) revisión crítica del manuscrito.

P. R. O. ha contribuido en a, c, d, e; E. S. E. en c, d, e; B. C. D. en b, c, d.

Editora científica responsable: Mag. Florencia de León 